EPJ Web of Conferences 88, 00027 (2015)

DOI: $10.1051 /$ epjconf/20158800027

(C) Owned by the authors, published by EDP Sciences - SIF, 2015

\title{
Isospin effects in heavy-ion reactions: Results from transport theories
}

\author{
M. Colonna ${ }^{1}$, V. BARAN ${ }^{2}$ and C. Rizzo ${ }^{1}$
}

${ }^{1}$ INFN-Laboratori Nazionali del Sud, I-95123, Catania, Italy

${ }^{2}$ NIPNE-HH, Bucharest and Bucharest University, Romania

\begin{abstract}
We discuss recent studies, within the framework of transport theories, on heavy ion reactions between charge asymmetric systems, from low up to Fermi energies. We focus on isospin sensitive observables, aiming at extracting information on the density dependence of the isovector part of the nuclear effective interaction and of the nuclear symmetry energy. Results are critically reviewed, also trying to establish a link, when possible, between the outcome of different transport models.
\end{abstract}

\section{Introduction}

The behavior of nuclear matter in several conditions of density, temperature and $\mathrm{N} / \mathrm{Z}$ asymmetry is of fundamental importance for the understanding of many phenomena involving nuclear systems and astrophysical compact objects. This information can be accessed by mean of heavy ion collision experiments, where transient states of nuclear matter spanning a large variety of regimes can be created. Actually this study allows one to learn about the corresponding behavior of the nuclear effective interaction, which provides the nuclear Equation of State (EOS) in the equilibrium limit. In particular, many investigations are concentrated nowadays on the EOS of asymmetric matter (Asy-EOS), which has, comparatively to the symmetric EOS [1], few experimental constraints. 
We stress that the latter information is essential for the understanding of some important properties of neutron stars, whose crust behaves as lowdensity asymmetric nuclear matter [2] and whose core may touch extreme values of density and asymmetry. Moreover, the low-density behavior of the symmetry energy also affects the structure of exotic nuclei and the appearance of new features involving the neutron skin, whish are currently under intense investigation [3].

Over the past years, several observables which are sensitive to the AsyEOS and testable experimentally, have been suggested, see ref. [4] for a recent review. In this article we will present some recent transport theory results on dissipative collisions, in a range of beam energies from just above the Coulomb barrier up to the Fermi regime.

\section{Transport theories and symmetry energy}

Nuclear reactions are modeled by solving transport equations based on mean field theories, with short range $(2 \mathrm{p}-2 \mathrm{~h})$ correlations included via hard nucleon-nucleon elastic collisions and via stochastic forces, selfconsistently evaluated from the mean phase-space trajectory, see [5].

In the energy range up to a few hundred $A M e V$, the appropriate tool is the so-called Boltzmann-Langevin (BL) equation [6]:

$$
\frac{d f}{d t}=\frac{\partial f}{\partial t}+\{f, H\}=I_{\text {coll }}[f]+\delta I[f]
$$

where $f(\mathbf{r}, \mathbf{p}, t)$ is the one-body distribution function, the semi-classical ana$\log$ of the Wigner transform of the one-body density matrix, $H(\mathbf{r}, \mathbf{p}, t)$ the mean field Hamiltonian, $I_{\text {coll }}$ the two-body collision term incorporating the Fermi statistics of the particles, and $\delta I[f]$ its fluctuating part.

Several approximate treatments of the BL equation have been introduced so far, denoted as one-body (OB) models in the following. For instance, in BUU-like models, the fluctuating term $\delta I[f]$ is neglected [7], whereas in the Stochastic Mean Field (SMF) model [8] fluctuations are projected onto the coordinate space and are implemented agitating the spatial density profile.

In the molecular dynamics (MD) class of models, the many-body state is represented by a product (antisymmetrized or not) of single particle states, which are usually assumed to be gaussians, whose centroids are time dependent. Then the one-body density can be built, which follows a time evolution close to the one expressed by Eq.(1). However, the localization of the nucleon wave packects induces correlations in the propagation in the nuclear 


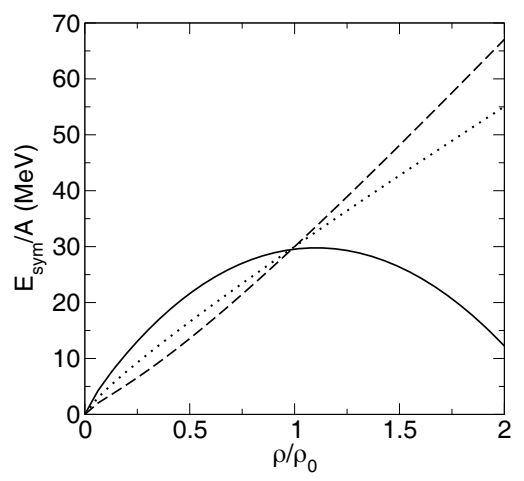

Figure 1: Three effective parameterizations of the symmetry energy : asystiff (dotted line), asysoft (full line) and asysuperstiff (dashed line).

mean-field, as well as in the collision integral, which is treated stochastically. Therefore, with respect to OB models, in MD models the fluctuation is expected to have a stronger impact on the collision dynamics, but some genuine "mean-field" effects could be missing, see $[9,10]$ and refs. therein.

Effective interactions (associated with a given EOS) can be considered as an input of all transport codes and from the comparison with experimental data one can finally get some hints on nuclear matter properties.

We recall that the symmetry energy $\frac{E_{s y m}}{A}=\frac{E_{s y m}}{A}(k i n)+\frac{C(\rho)}{2 \rho_{0}} \rho$ appears in the energy density functional $\epsilon\left(\rho, \rho_{i}\right) \equiv \epsilon(\rho)+\rho \frac{E_{\text {sym }}}{A}\left(\rho_{i} / \rho\right)^{2}+O\left(\rho_{i} / \rho\right)^{4}+$.., expressed in terms of total $\left(\rho=\rho_{p}+\rho_{n}\right)$ and isospin $\left(\rho_{i}=\rho_{p}-\rho_{n}\right)$ densities, from which the mean-field potential can be consistently derived $\left(\rho_{0}\right.$ denotes the saturation density). We adopt a soft isoscalar EOS (compressibility $K=200 \mathrm{MeV}$ ) and the free nucleon-nucleon cross section. The sensitivity of the simulation results is tested against different choices of the density dependence of the isovector part of the effective interaction. For instance, the symmetry energy behavior associated with three different parameterizations of $C(\rho)$ (the asysoft, the asystiff and asysuperstiff) is displayed in fig.1, see [5] for a detailed description.

\section{Fragmentation at low energy: Ternary breakup}

We discuss semi-peripheral heavy ion collisions in the beam energy range of $10-30 \mathrm{MeV} /$ nucleon, focusing on the possible occurrence of ternary breakup processes and on the features of the associated reaction products. Recent experimental investigations have been concentrated on these exotic separation modes, in the case of $\mathrm{Au}+\mathrm{Au}$ reactions at 15 and $23 \mathrm{MeV} / \mathrm{A}[11,12]$.

Let us consider first the reaction at $15 \mathrm{MeV} / \mathrm{A}, \mathrm{b}=[4-6] \mathrm{fm}$, in the 


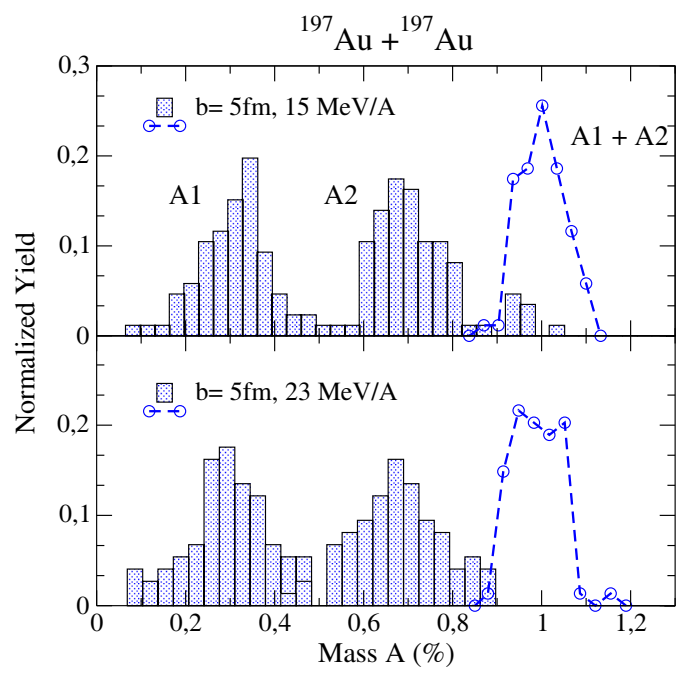

Figure 2: Mass distribution of the fragments A1 and A2, and of their sum, emerging from the breakup of the PLF/TLF fragment, for the reaction ${ }^{197} \mathrm{Au}+$ ${ }^{197} \mathrm{Au}$ at 15 (top) and 23 (bottom) $\mathrm{MeV} / \mathrm{A}$. A1 denotes the smallest fragment. Masses are normalized to the average mass of the PLF/TLF fragment.

framework of SMF simulations. In this case we observe an almost symmetric neck rupture between PLF and TLF, leading to the formation of two deformed fragments in the majority of the events. Important quadrupole and octuple deformations are noticed for the primary PLF/TLF fragments, which may lead to subsequent ruptures (multiple breakup) and, in particular, to ternary breakup events. An early recognition method is applied to identify the most probable breakup configuration of the PLF (or TLF) fragment.

Figure 2 (top panel) shows the mass distribution of the lightest, A1, and heaviest, A2, fragments for the impact parameter $\mathrm{b}=5 \mathrm{fm}$. Once the masses are normalized to the average mass of the PLF (or TLF) fragment, results look in good agreement with experimental data (see ref. [11]). In particular, the calculations are able to reproduce the distance between the A1 and A2 centroids and the variance of the mass distribution. Similar results have been recently reported in the context of the improved QMD (ImQMD) calculations of ref. [10], where a detailed comparison with the experimental findings of ref. [11] is presented. Results obtained at the higher bombarding energy of $23 \mathrm{MeV} / \mathrm{A}$ are shown in the bottom panel of the figure. The behavior observed for the mass distribution of fragments A1 and A2 is quite close to the one obtained at $15 \mathrm{MeV} / \mathrm{A}$, however variances are larger, reflecting the more dissipative dynamics.

It should be noticed that, in the calculations of ref. [10], as well as in SMF simulations at $15 \mathrm{MeV} / \mathrm{A}$, the lightest fragment (A1) emerges mainly from the neck region, thus being located at mid-velocity. On the other 
hand, in the data analysis reported in ref. [12], the fragment with the largest parallel velocity (F1) has the smallest mass. In SMF simulations, this feature is present, but at higher energy. Indeed, at $23 \mathrm{MeV} / \mathrm{A}$, owing to increased angular momentum effects, the PLF (or TLF) fragment rotates before it reaches its maximum deformation and a subsequent breakup may take place. As a consequence, in the case of a PLF break-up for instance, the lightest fragment may emerge with large positive parallel velocity. Thus SMF results point to the occurrence of a reaction mechanism, i.e. neck rupture coupled to angular momentum effects, which could explain the experimental observation. On the other hand, in QMD-like calculations rotational effects could be missing because of the too fast reaction dynamics and the reduced mean-field effects [9].

In the calculations discussed above, the sensitivity to the symmetry energy parametrization has not been explored so far. It would be extremely interesting to extend this kind of investigations to reactions involving neutronrich (or even exotic) nuclei [13]. Indeed the reaction dynamics could be affected by the neutron-enrichment of the neck region, related to neutron skin effects and/or isospin migration mechanisms $[5,14]$. In this case, one would also expect an important sensitivity of the reaction mechanism, and of the features of the emitted fragments, to the isovector terms of the nuclear potential, opening interesting perspectives towards the extraction of new, independent information on the density behavior of the nuclear symmetry energy [4].

\section{Isovector fluctuations and isoscaling}

Let us move to discuss the possibility to extract information on the lowdensity behavior of the symmetry energy from the isotopic features of multifragmentation processes. The best suited framework to explore this subject is to consider nuclear matter in a box. Calculations are performed taking, as initial density $\rho_{i n}$, three values inside the spinodal region: $\rho_{1}=0.0245 \mathrm{fm}^{-3}$, $\rho_{2}=2 \rho_{1}$ and $\rho_{3}=3 \rho_{1}$. Moreover, for each case, we have considered symmetric matter (system (1), $I_{1}=0$ ) and asymmetric matter (system (2), $\left.I_{2}=0.142\right)[15]$.

The unstable system will develop density fluctuations, so locally the density gets larger (density bumps, leading to fragments) or smaller (vapour) than $\rho_{\text {in }}$ [5]. Our aim is to investigate the behavior of isovector fluctuations on the short time scale (the "freeze-out" time) associated with fragment formation. Isovector fluctuations are evaluated as a function of the local 


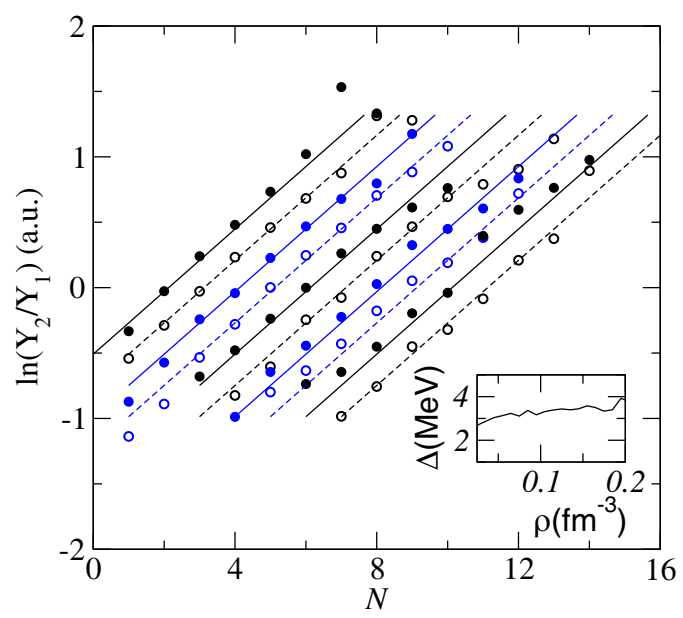

Figure 3: (Color online) The quantity $\ln \left(Y_{2} / Y_{1}\right)$ is plotted as a function of $N$, for the charges $Z=1-10$, in the case of the systems with initial density $\rho_{1}$. The asystiff parametrization in considered. Lines are to guide the eye. The inset shows the product $\Delta=4 \Delta(\overline{Z / A})^{2} \cdot F^{s y m}$, as a function of the local density.

density inside the fragmenting system, looking at the variance of the isovector density $\rho^{v}=\rho_{n}-\rho_{p}$ in cells, of volume $\Delta V$, having the same local density $\rho$. As a measure of the isovector variance $\sigma_{\rho^{v}}$, we consider the quantity $F_{u n s}^{v}=(\rho T) /\left(2 \Delta V \sigma_{\rho^{v}}\right)$, that coincides with the free symmetry energy, $F^{s y m}$ if equilibrium is reached. As shown in ref. [15], we observe that isovector fluctuations follow the local value of the symmetry energy, reflecting the parametrization (stiff or soft) considered, independently of the initial conditions of the system. Indeed the quantity $F_{\text {uns }}^{v}$ is close for each given local density, to the equilibrium results, thus locally $F_{u n s}^{v} \approx F^{s y m}$. These results indicate that, as soon as density fluctuations start to develop, a quick rearrangement of isovector fluctuations takes place, so that the equilibrium value corresponding to the new actual local density is approached. In the case of the asymmetric system (2) one also observes the isospin distillation mechanism, that induces a deviation of the local asymmetry from the system initial value [5].

Let us move to study the probability $Y(Z, N)$ to find, inside a volume $V$, a given number of protons and neutrons, $Z=\rho_{n, V} V$ and $N=\rho_{p, V} V$. $\rho_{n, V}$ and $\rho_{p, V}$ denote neutron and proton densities averaged over $V$, whose sum yields the density $\rho_{V}$. We consider $V=(5.5 \mathrm{fm})^{3}$. The quantity $Y(Z, N)$ is proportional to the probability of getting, in the volume $V$, a specific variation of the isovector density $\rho^{v}$, with respect to the average $\overline{\rho^{v}}$ : $P\left(\rho^{v}\right) \approx \exp -\left(\rho^{v}-\overline{\rho^{v}}\right)^{2} /\left(2 \sigma_{\rho^{v}}\right)$. Using the identity $\rho^{v} / \rho_{V}=I=(N-Z) / A$ and considering the equilibrium amplitude of $\sigma_{\rho^{v}}$, one can write, for the yield ratio between systems (1) and (2):

$$
\ln \left(Y_{2} / Y_{1}\right) \approx\left[\left(I-\bar{I}_{1}\right)^{2}-\left(I-\bar{I}_{2}\right)^{2}\right] A F^{s y m} / T .
$$


After some algebra, expressing $\bar{I}_{i}$ in terms of the average proton or neutron fraction, we get: $\ln \left(Y_{2} / Y_{1}\right) \approx \alpha N+\beta Z$, with:

$$
\begin{aligned}
& \alpha\left(\rho_{V}\right)=4 \Delta(\overline{Z / A})^{2} F^{s y m} / T, \\
& \beta\left(\rho_{V}\right)=4 \Delta(\overline{N / A})^{2} F^{s y m} / T .
\end{aligned}
$$

Thus we recover the standard isoscaling relations [16], but with densitydependent coefficients $\alpha\left(\rho_{V}\right)$ and $\beta\left(\rho_{V}\right)$, linked to the local symmetry free energy $F^{\text {sym }}$ and local proton fraction.

The behavior of the exponent $\alpha$ is illustrated in fig.3, where we plot the quantity $\ln \left(Y_{2} / Y_{1}\right)$ as a function of $N$, for the charges $Z=1-10$. Thus our dynamical analysis confirms that fragment isospin fluctuations and isoscaling parameters are related to the symmetry energy at the fragment formation density. These results are relevant to experimental isoscaling analyses aiming at extracting information on the symmetry energy [17]. Recently a collection of these experimental results has been published in ref. [18]. It is interesting to see how the different experiments give quite close results as far as the extraction of the symmetry energy, as a function of the excitation energy of the system, is concerned. The dynamical study discussed above provides a theoretical background to these experimental investigations.

\subsection{Isospin equilibration: Comparison between the predic- tions of different transport codes}

A detailed investigation of the degree of charge equilibration reached in semiperipheral collisions at Fermi energies has been recently undertaken within transport codes based on the molecular dynamics (MD) approach [19]. For $S n+S n$ reactions at 35 and $50 \mathrm{MeV} / u$, the ImQMD code predicts a quite different behavior with respect to SMF: the isospin transport ratio exhibits a rather flat behavior as a function of the impact parameter. This seems to indicate that, even in the case of central collisions, the contact time between the two reaction partners remains rather short, the dissipation mechanisms being mostly due to many-body correlations rather than to mean-field effects. Thus the more explosive dynamics, characterized by cluster and intermediate mass fragment (IMF) emission, would lead to the lower degree of isospin equilibration observed.

Isospin diffusion has been recently investigated also in the context of a BUU approach including cluster production, the pBUU model [20]. It is found that cluster production reduces diffusion and causes the neck to become more isospin symmetric [21]. Thus it is clear that cluster production has an important influence on the charge equilibration process. 

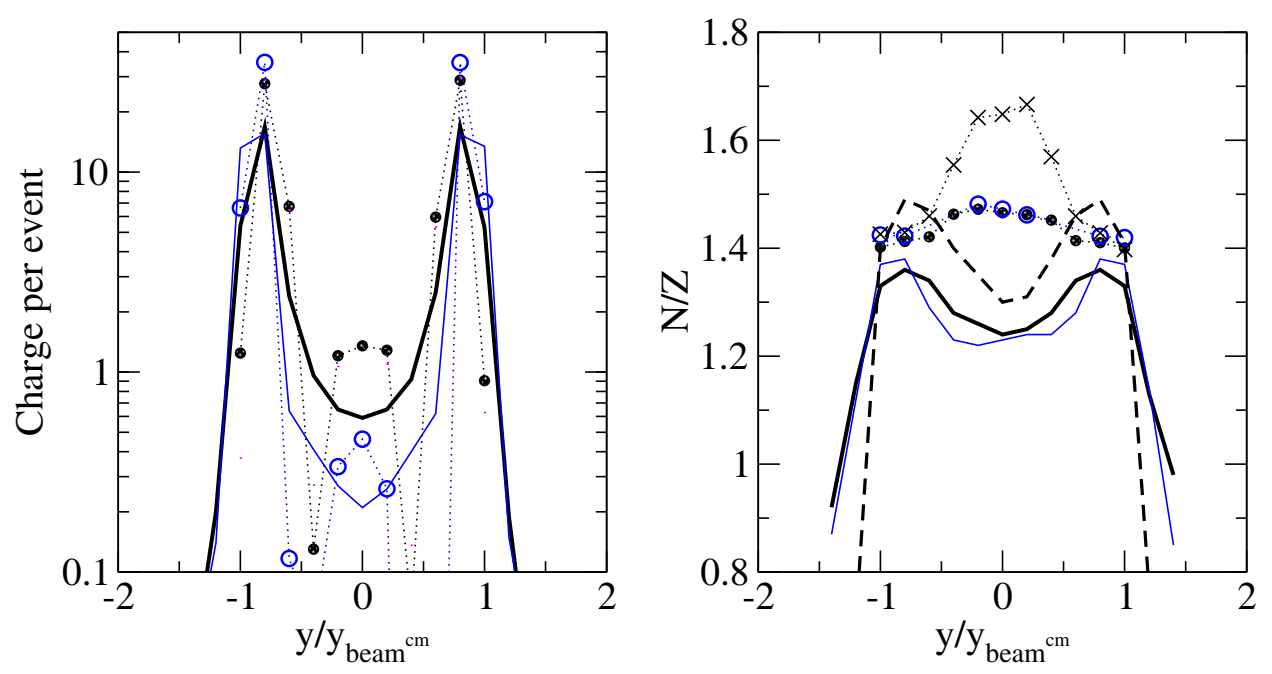

Figure 4: Let panel: Average total charge per event, associated with IMF's, as a function of the reduced rapidity, obtained in the reaction ${ }^{124} \mathrm{Sn}+{ }^{124} \mathrm{Sn}$ at 50 $\mathrm{MeV} / \mathrm{u}$. Results are shown for ImQMD calculations at $\mathrm{b}=6 \mathrm{fm}$ (thick line) and $\mathrm{b}=8 \mathrm{fm}$ (thin line) and for SMF calculations at $\mathrm{b}=6 \mathrm{fm}$ (full circles) and $\mathrm{b}=8$ $\mathrm{fm}$ (open circles). A soft interaction is considered for the symmetry energy. Right panel: N/Z of IMF's as a function of the reduced rapidity. Lines and symbols are like in the left panel. Results corresponding to a stiff asy-EOS are also shown for ImQMD (dashed line) and SMF (crosses), for $\mathrm{b}=6 \mathrm{fm}$.

To examine more in detail the discrepancies observed between the transport models considered, results concerning IMF $(Z>2)$ properties, obtained with the SMF and ImQMD codes, are compared in figure 4 . In the left panel, the average total charge per event, associated with IMF's, is plotted as a function of the reduced rapidity, for the reaction ${ }^{124} \mathrm{Sn}+{ }^{124} \mathrm{Sn}$ at 50 $\mathrm{MeV} / \mathrm{u}$ and impact parameters $\mathrm{b}=6$ and $8 \mathrm{fm}$. From this comparison it is clear that in ImQMD a larger number of light IMF's, distributed over all rapidity range between PLF and TLF, are produced. On the other hand, mostly binary or ternary events are observed in SMF, with IMF's located very close to mid-rapidity. Then the different reaction dynamics predicted by the two codes may explain the different results seen for isospin equilibration especially in semi-peripheral and central reactions $(b \approx 4-6 \mathrm{fm})$. The fast ImQMD fragmentation dynamics inhibits nucleon exchange and charge equilibration. On the other hand, in SMF dissipation is dominated by meanfield mechanisms, acting over longer time intervals and leading to stronger equilibration effects. The different reaction dynamics has an impact also on 
the neutron content of the neck region. Corresponding results are illustrated in the right panel of figure 4, that shows the global N/Z of IMF's as a function of the reduced rapidity. SMF calculations clearly predict a larger $\mathrm{N} / \mathrm{Z}$ for IMF's produced at mid-rapidity, with respect to PLF and TLF regions (isospin migration effect). The effect is particularly pronounced in the case of the asystiff parametrization. On the contrary, ImQMD calculations predict a minimum of the $\mathrm{N} / \mathrm{Z}$ ratio at mid-rapidity. Also in the framework of the pBUU model, owing to the emission of neutron-rich clusters, the $\mathrm{N} / \mathrm{Z}$ content of the neck region is reduced [21].

The delicate balance between mean-field and correlation effects emerges as an important issue to be investigated in the future. Indeed, from one side cluster emission reduces the degree of charge equilibration, in the direction of the experimental results [19]. However, the effect of neutron enrichment of the neck region, which has been experimentally reported in recent works [14], is not reproduced by the available approaches including cluster emission. On the other hand, the latter effect is reproduced by OB models, like SMF [14].

\section{Conclusions}

In this contribution we have reviewed some results concerning fragmentation and charge equilibration mechanisms at low and Fermi energies. We have investigated the probability of observing ternary breakup modes in semiperipheral reactions at $\approx 20 \mathrm{MeV} / \mathrm{A}$, which is expected to be sensitive to the symmetry energy parameterization in collisions between n-rich systems. Isotopic features of multi-fragmentation processes have been analysed in the framework of transport theories, showing that equilibrium is quickly reached for isovector density fluctuations. Thus the fragment isotopic variances reflect the local value of the symmetry energy, supporting the experimental analyses aiming at extracting the latter information from isoscaling observables. Finally, we have discussed the degree of charge equilibration reached in reactions at Fermi energies, comparing the results of different transport theories. Further investigations are needed to elucidate the respective role of mean-field vs. correlation effects.

\section{Acknowledgements}

This work, for V. Baran, was supported by a grant of the Romanian National Authority for Scientific Research, CNCS - UEFISCDI, project number PNII-ID-PCE-2011-3-0972. 


\section{References}

[1] Danielewicz P., Lacey R. and Lynch W.G., Science 298 (2002) 1592.

[2] Lattimer J.M and Prakash M., Phys. Rep. 442 (2007) 109.

[3] Piekarewicz J. et al., Phys Rev C 85 (2012) 041302.

[4] Topical Issue on Nuclear Symmetry Energy, Eur. Phys. Jou. A50 (2014), edited by B-A Li, A. Ramos, G. Verde and I. Vidana.

[5] Baran V., Colonna M., Greco V. and Di Toro M., Phys. Rep. 410 (2005) 335 .

[6] Ayik S., Gregoire C., Phys. Lett. B 212 (1988) 269.

[7] Li B-A., Das C.B., Das Gupta S. and Gale C., Phys. Rev. C 69 (2004) 011603(R).

[8] Colonna M. et al., Nucl. Phys. A 642 (1998) 449.

[9] Colonna M., Ono A., Rizzo J., Phys. Rev. C 82 (2010) 054613.

[10] Li Y., Yan S., Jiang X., Wang L., Nucl. Phys. A 902 (2013) 1.

[11] Skwira-Chalot I. et al., Int. Jou. of Mod. Phys. E 15, (2006) 495.

[12] Wilczynski J. et al., Phys. Rev. C 81 (2010) 024605.

[13] Cammarata P. et al., NIM A 761 (2014) 1.

[14] De Filippo E. et al. (Chimera Collab.), Phys. Rev. C 86 (2012) 014610.

[15] Colonna M., Phys. Rev. Lett. 110 (2013) 042701.

[16] Botvina A. et al., Phys. Rev. C 65 (2002) 044610.

[17] Tsang M.B. et al., Phys. Rev. Lett. 86 (2001) 5023.

[18] Kohley Z.,Yennello S. J., Eur. Phys. Jou. A 50 (2014) 31.

[19] Tsang M.B. et al, Phys. Rev. Lett. 102 (2009) 122701; Sun Z.Y. et al,, Phys. Rev. C 82 (2010) 051603.

[20] Danielewicz P. and Bertsch G.F., Nucl. Phys. A 533 (1991) 712.

[21] Coupland D. et al., Phys. Rev. C 84 (2011) 054603. 